\title{
CLAVES PEDAGÓGICAS PARA LA RÚBRICA DE APRENDIZAJE - SERVICIO
}

\author{
Yolanda Ruiz Ordóñez \\ Universidad Católica de Valencia \\ yolanda.ruiz@ucv.es
}

Fecha de Recepción: 12 Febrero 2019

Fecha de Admisión: 30 Abril 2019

\section{RESUMEN}

Son diversas las rúbricas de evaluación para los proyectos de aprendizaje-servicio que están presenten en el ámbito académico. Cada una pone el énfasis en unos aspectos concretos respondiendo a la finalidad del mismo. Atendiendo a la preocupación por disponer de una herramienta evaluativa en esta materia que respondiera a la educación integral de los alumnos y valorando las interesantes aportaciones del Papa Francisco en materia de educación, se ha realizado un rastreo exhaustivo de sus escritos. Desde un análisis de los mismos, se han propuesto las claves educativas de su pedagogía que servirán como fundamento para la elaboración de una rúbrica evaluativa de los proyectos de Aprendizaje-Servicio.

Una línea fundamental se basa en comprender estos proyectos como una oportunidad para humanizar la educación, destacando pues factores antropológicos y existenciales como el desarrollo de la interioridad y de la dimensión espiritual-personal. El educador es, por tanto, un guía que ayuda al crecimiento personal y madurativo de los alumnos.

El objetivo del presente trabajo es presentar una Rúbrica para la Evaluación de Experiencias de Aprendizaje Servicio con el fin de promover la capacitación y el desarrollo integral del alumno. Ésta se estructura en dos ejes que se cruzan: persona y contexto. Comprende seis dimensiones: cultura, educación, agentes sociales, aprendizaje, valores y servicio. Cada dimensión evalúa 5 categorías pedagógicas que son evaluados en 4 niveles. Herramientas de este tipo permiten motivar en conductas prosociales a los alumnos para que sean agentes de transformación social y desarrollen una ciudadanía activa que trabaje por una sociedad más justa y pacífica.

Palabras clave: aprendizaje; servicio; educación integral; prosocialidad

\section{ABSTRACT}

Pedagogical keys for learning service rubrics. There are several assessment rubrics for service-learning projects that are presented in the academic field. Each one puts the emphasis on some 
specific aspects responding to its purpose. Attending to the concern for having an evaluation tool in this area that would respond to the integral education of the students and valuing the interesting contributions of Pope Francisco in the matter of education, an exhaustive tracking of his writings has been carried out. From an analysis of them, the educational keys of their pedagogy have been proposed that will serve as the basis for the elaboration of an evaluative rubric of the LearningService projects.

A fundamental line is based on understanding these projects as an opportunity to humanize education, thus highlighting anthropological and existential factors such as the development of interiority and the spiritual-personal dimension. The educator is, therefore, a guide that helps the personal growth and maturation of the students.

The objective of this paper is to examine a Rubric for the assessment of Service Learning Experiences in order to promote training and the integral development of the student. This is structured into two intersecting axes: person and context. It comprises six dimensions: culture, education, social agents, learning, values and service. Each dimension evaluates 5 pedagogical categories that are evaluated in 4 levels. Tools of this type allow students to be motivated in prosocial behavior. Thus, they are agents of social transformation and develop an active citizenship that works for a more just and peaceful society.

Keywords: service learning; integral education; prosociality

\section{LA EDUCACIÓN EN FRANCISCO}

Para el Papa Francisco la educación está muy ligada al proceso de humanización y por tanto, a aspectos antropológicos y existenciales. Esta forma de concebir la educación implica un modo de transmisión de la cultura, sabiendo que la crisis cultural y de valores están presentes en la sociedad. En este sentido, el entonces cardenal Bergoglio proponía la necesidad de realizar un discernimiento cultural, pues actualmente las estructuras predominan sobre el ethos, y los valores quedan totalmente desligados de las raíces del pensamiento cristiano. Fruto de un mesianismo profano y de un relativismo en el que los valores son resultados de pactos, en la educación subyace una antropología gnóstica o carente de unidad y esotérica o secularizada, dejando que la mentalidad tecnicista impregne el paradigma educativo. Ante esta situación, la propuesta es promover la cultura del encuentro y desde ella crear vínculos que no rechacen a nadie, especialmente al hombre sufriente. Esto implica que los educadores generemos espacios de encuentro desde la autoridad que tenemos. Nuestros jóvenes poseen unos modos de pensar, sentir y actuar; los educadores debemos saber qué aspectos les preocupan y ayudarles a interpretar el mundo que les rodea (Bergoglio, 1999).

En el Curso de Rectores, Bergoglio (2006) entendía que hablar de hecho educativo es hacerlo de encuentro educativo donde la interioridad y la dimensión espiritual-personal son especialmente importantes. El educador no es un técnico, más bien es quien debe extraer de dentro y ayudar a hacer crecer. Afirmar el carácter técnico del docente es negar la existencia de un encuentro educativo. Para Francisco educar no sólo es transmitir conocimientos, sino hábitos y valoraciones que conducen al modo de actuar o situarse en el mundo. Para ello, el joven debe ser utópico y tener presente la memoria del pasado y discernimiento para su momento actual. De esta manera es posible caminar hacia el horizonte del futuro (Francisco PP, 2014c). La educación trasciende lo que es la adquisición de conocimientos técnicos o competencias, pues lo que debe de fomentar es el crecimiento y madurez de las personas. Así, los jóvenes podrán tener una formación que les oriente y mantenga viva su esperanza hacia el futuro (Francisco PP, 2014f).

Actualmente, los educadores hemos de afrontar grandes retos. Los cambios de la sociedad actual exigen cambios en el modo de educar. Francisco sostiene que para que la educación avance, 
el alumno debe de pisar firme en lo adquirido porque le hace sentirse cómodo y seguro, pero también dirigirse hacia la zona de riesgo, valorando que ambas zonas, seguridad y riesgo, sean proporcionales a la persona y al entorno (Rubin \& Ambrogetti, 2015, p.61) Por eso, hay que educar en un equilibrio entre la zona de riesgo y la zona de seguridad, ya que es de este modo cómo crecen las personalidades. Estos aspectos hay que tenerlos en cuenta en todo este proceso que pretenda educar a la persona, así como el lugar, los tiempos y el contexto (Francisco PP, 2013a).

En el viaje apostólico a Río de Janeiro con ocasión de la XXVIII Jornada Mundial de la Juventud, el Santo Padre afirmaba que:

la juventud es el ventanal por el que entra el futuro en el mundo. Es el ventanal y, por tanto, nos impone grandes retos. Nuestra generación se mostrará a la altura de la promesa que hay en cada joven cuando sepa ofrecerle espacio. Esto significa tutelar las condiciones materiales y espirituales para su pleno desarrollo; darle una base sólida sobre la que pueda construir su vida; garantizarle seguridad y educación para que llegue a ser lo que puede ser; transmitirle valores duraderos por los que valga la pena vivir; asegurarle un horizonte trascendente para su sed de auténtica felicidad y su creatividad en el bien; dejarle en herencia un mundo que corresponda a la medida de la vida humana; despertar en él las mejores potencialidades para ser protagonista de su propio porvenir, y corresponsable del destino de todos (Francisco PP, 2013b).

La educación no puede ser neutra sino que debe de ayudar al joven a desarrollar una serie de actitudes y valores, enseñándole también a pensar de forma crítica (Francisco PP, 2013c). Los educadores necesitan saber comunicarse con los jóvenes. No es suficiente ser altamente cualificado y tener un estilo pedagógico. Para que los jóvenes adquieran actitudes y valores, éstos deben ser testimoniados y no referenciados. De ahí que la coherencia y el testimonio son rasgos claves de la figura del educador. "Educar es un acto de amor, es dar vida. Y el amor es exigente, pide utilizar los mejores recursos, despertar la pasión y ponerse en camino con paciencia junto a los jóvenes" (Francisco PP, 2014b).

\section{LA RÚBRICA PARA LA EVALUACIÓN DE EXPERIENCIAS DE APRENDIZAJE-SERVICIO}

La Rúbrica para la Evaluación de Experiencias de Aprendizaje-Servicio se elabora con el fin de que los docentes e instituciones educativas que deseen aplicar dicha metodología, dispongan de un instrumento que permita valorar si los proyectos están en coherencia y consonancia con los criterios pedagógicos del Papa Francisco. Entre los elementos principales que prevalecen están la cultura del encuentro y la reconstrucción del pacto educativo. En la sociedad actual, impregnada de conflictos y desencuentros, la cultura del encuentro es un desafío. Además, el Santo Padre nos propone "recomponer el pacto educativo" para que el educando no se quede solo, aislado y vulnerable, sino que se le acompañe en todo momento con la finalidad de que sea parte activa de una sociedad. Y para lograrlo, es necesario fortalecer los vínculos sociales, familiares y personales, desarrollando armónicamente un entorno adecuado, creándose lazos que abran a la realidad y que sean lugar de encuentro con lo bueno, verdadero y bello. Es ahí donde reside la esperanza de la educación (Francisco PP, 2014e). En consecuencia, para poder restaurar el pacto educativo, la familia, el Estado, los docentes y las diferentes confesiones religiosas deben estar alineadas en una acción concertada (Rubin \& Ambrogetti, 2015, p. 66).

Independientemente de las diferencias presentes en cada alumno, los educadores hemos de formar a nuestros estudiantes para que sean agentes activos de transformación social y trabajen por el bien común, la solidaridad y la paz, rechazando cualquier tipo de discriminación e intolerancia. Es necesario que los alumnos abran los ojos y tomen conciencia del mundo que les rodea y, de esta manera, puedan sentirse actores sociales principales, capaces de cambiar la realidad. 
Desgraciadamente, nuestra sociedad del bienestar está excesivamente apoyada en la posesión y el disfrute de bienes materiales, cerrando la posibilidad a las personas de percibir necesidades ajenas. Hay que romper esa falta de sensibilidad. Por eso, la educación debe promover que los agentes educativos salgan al encuentro del otro y eviten, de esta manera, cualquier modo de autorreferencialidad para dejarse interpelar y actuar sobre la realidad. Es entonces cuando las instituciones educativas hemos de ofrecer métodos pedagógicos que permitan desarrollar actitudes de diálogo, de comprensión y de empatía.

La educación ha de contemplar también espacios de promoción integral de la persona en todas sus dimensiones sin eliminar a nadie, rompiendo prejuicios y acogiendo la vulnerabilidad y necesidad. Hay que trabajar por un mundo más justo, más fraterno y en paz. Tener una visión educativa de este tipo permite a todos los agentes educativos, ya sean alumnos, ya sean profesores, una mayor comprensión del mundo que nos rodea, al mismo tiempo que se genera un nuevo estilo de vida, porque se trabajan actitudes incidiendo sobre la interacción y coherencia entre la dimensión afectiva 0 el sentir (lenguaje del corazón), la cognitiva o el pensar (lenguaje de la cabeza) y la conducta 0 el actuar (lenguaje de las manos) (Rubin \& Ambrogetti, 2015, p. 62).

El Santo Padre Francisco (2014, 10 de mayo) en el discurso al mundo de la escuela italiana en la Plaza de San Pedro afirmaba que "la escuela es un lugar de encuentro" donde cada uno nos encontramos con los compañeros, profesores, alumnos y personal no docente. Para seguir abriendo caminos necesitamos desarrollar esta cultura del encuentro que, en definitiva, es la que permite socializarnos e integrarnos en la sociedad. La escuela y la universidad deben educar en la verdad, y permitir que la persona crezca y se enriquezca. El Papa Francisco, en este mismo discurso a la escuela italiana, añade que ofreciendo una educación buena:

cultivamos en nosotros lo verdadero, el bien y lo bello; y aprendemos que estas tres dimensiones no están jamás separadas, sino siempre entrelazadas. Si una cosa es verdadera, es buena y es bella; si es bella, es buena y es verdadera; y si es buena, es verdadera y es bella. Estos elementos juntos nos hacen crecer y nos ayudan a amar la vida, incluso cuando estamos mal, también en medio de los problemas. La verdadera educación nos hace amar la vida y nos abre a la plenitud de la vida (Francisco PP, 2014d).

Se trata pues, como sigue afirmando en dicho discurso, de:

un camino que haga crecer las tres lenguas que una persona madura debe saber hablar: la lengua de la mente, la lengua del corazón y la lengua de las manos. Pero con armonía, es decir, pensar lo que tú sientes y lo que tú haces; sentir bien lo que tú piensas y lo que tú haces; y hacer bien lo que tú piensas y lo que tú sientes. Las tres lenguas, armoniosas y juntas (Francisco PP, 2014d).

Las dimensiones cognitivas y afectivas deben integrarse armónicamente para poder articular la conductual, generando un estilo de vida.

Desde este contexto y teniendo presente la importancia de promover metodologías docentes activas surge la metodología Aprendizaje-Servicio que permite al alumno adquirir competencias curriculares interviniendo sobre la realidad al mismo tiempo que se presta un servicio a la comunidad. Esta metodología pedagógica ayuda a desarrollar actitudes de diálogo, de comprensión y de empatía; y como afirma Palos:

conlleva de forma inmediata un cambio en la forma de trabajar del profesorado que a largo plazo se tiende a consolidar...estas actividades inducen al profesorado y a otros profesionales a abrirse al entorno físico, social y cultural donde se encuentran los centros (Puig y otros, 2009, p. 156).

Aportaciones como la de Tapia, que se sirve la definición de J. Kielsmeier, aseguran que con Aprendizaje Servicio, los alumnos son ciudadanos activos de la comunidad. Se concibe como un modelo de desarrollo comunitario, ya que guarda una relación directa con necesidades y problemas 
reales. Es un método de enseñanza-aprendizaje desde el que el alumno educa el pensamiento crítico, así como la capacidad de resolver problemas. Por eso, no únicamente mejora la participación ciudadana y personal, sino también el rendimiento académico (Tapia, 2004, p.15).

Los educadores hemos de innovar y de proponer metodologías que permitan entrar en diálogo con los jóvenes, considerando sus inquietudes, desarrollando acciones en contextos reconocibles para ellos y motivando para que sean los protagonistas de su propio aprendizaje. Al mismo tiempo, tenemos que sensibilizarles para que sean partícipes y actúen ante realidades injustas que no conciben a la persona como fin. Se trata de humanizar la educación, acompañando procesos y relaciones que ayuden a despertar las conciencias de los agentes educativos a favor del bien común. Metodologías como Aprendizaje Servicio se centran en el aprendizaje del estudiante y atienden a las necesidades reales para intervenir y mejorarlo. Además, dicha propuesta de mejora es planteada por el alumno que es quien aprende observando, conociendo, reflexionando y respondiendo a una necesidad social. "Aprendizaje-Servicio es un método para aprender a participar en la vida social" (Puig, Batlle, Bosch, y Palos, 2007, p. 37)

Los proyectos de Aprendizaje-Servicio no son prácticas de voluntariado aisladas del conjunto de conocimientos y competencias que procuran asumir los distintos planes docentes de una titulación, más bien son propuestas docentes vinculadas curricularmente a las asignaturas y orientadas a una participación activa. Son ejemplos visibles de una pedagogía de la experiencia.

Los jóvenes aprenden a observar de manera crítica su entorno, a detectar problemas, a desarrollar la sensibilidad para sentirse afectados por las necesidades ajenas, y, por último, entrenan y desarrollan capacidades que ponen al servicio del bien común: la autonomía, la iniciativa personal y el talante emprendedor están muy presentes en una pedagogía que defiende la experiencia como elemento insustituible del proceso formativo (Puig y otros, 2009, p. 109).

Así pues la Rúbrica de experiencias de Aprendizaje Servicio se convierte en una herramienta muy útil para los profesionales de la educación que quieren aplicar este método innovador y evaluar la coherencia de dichos proyectos desde una perspectiva educativa sustentada en la pedagogía de Scholas Occurrentes. Es necesario advertir que lo que se propone a continuación no es garantía exclusiva de una cambio actitudinal en el alumno tras la aplicación de esta metodología porque influyen, probablemente, otros factores difícilmente detectables y controlables. Sin embargo, sí que consideramos que es un recurso que ayuda y contribuye a sensibilizar en el cambio de valores y actitudes.

\section{ESTRUCTURA DE LA RÚBRICA}

Para proceder a elaborar la Rúbrica, se ha realizado un rastreo de literatura del Papa Francisco seleccionando aquellas ideas y conceptos que guardan estrecha relación en materia de educación. El paradigma bajo el que establecemos los ejes es el pacto educativo. Es necesario recomponerlo porque son numerosos los actores sociales que influyen en la educación. Los términos elegidos como cualidades para dicha rúbrica son los que aparecen en la Tabla 1 se han seleccionado a través del archivo digital de la web del Vaticano, en mayor número de veces en los discursos, homilías, cartas, mensajes, audiencias, cartas apostólicas y encíclicas del Papa Francisco elegidas para este propósito en lengua española. La suma total de veces que aparecen las voces o términos utilizados para la rúbrica desde los documentos anteriormente descritos del Papa Francisco alcanzan un total de 453.

Estas cualidades 0 actitudes que enmarcarán las dimensiones y categorías pedagógicas de la rúbrica son las que, en definitiva, pretendemos proponer desde la pedagogía del Papa Francisco. Cualidades que planteamos, además, como idóneas para evaluar los resultados en el alumno y su 
efecto en el entorno o contexto donde se desarrolla un proyecto de Aprendizaje-Servicio.

Desde esta perspectiva se han trazado dos ejes que dibujan 4 cuadrantes: persona en el eje vertical y entorno/contexto en el eje horizontal. El eje de persona es un continuo que se relaciona con la idea que el alumno sea capaz de encontrarse con el otro que es distinto a mí. Se vela para establecer vínculos personales y de trabajo. Encontrarnos con otro nos permite crecer y generar lazos, supone ser conscientes de que todos dependemos de todos y por eso, nuestra acción debe ser fruto del discernimiento. Si el alumno ha desarrollado una buena capacidad de encuentro con el otro situaremos el proyecto en el parte superior, y si por el contrario es menor la capacidad, lo haremos en la zona inferior. El segundo eje es el entorno/contexto que guarda relación con la idea de que cualquier modelo educativo tiene que ayudar al alumno a introducirse en el sentido de la realidad y a comprometerse con ella. Cuando el alumno y las experiencias educativas se implican con el entorno y con los actores sociales, es entonces cuando es posible actuar y transformar. Si el proyecto posee escaso compromiso con la realidad y por consiguiente menor capacidad de transformarla, lo situaremos en el extremo izquierdo y, si por el contrario, posee más compromiso, lo haremos en el derecho.

En cada una de los 4 espacios que se generan podemos encontrar 4 niveles:

Nivel l: cuando en el proyecto de Aprendizaje-Servicio, los estudiantes no se implican con la realidad del entorno porque no hay un cambio de actitudes que suponga un compromiso por el cambio del contexto. La adhesión a contribuir a rehacer el pacto educativo es baja.

Nivel Ila: cuando en el proyecto de Aprendizaje-Servicio, los estudiantes se implican con la realidad del entorno pero no hay un cambio de actitudes que suponga un compromiso por el cambio del contexto. La adhesión a contribuir a rehacer el pacto educativo es media.

Nivel IIb: cuando en el proyecto de Aprendizaje-Servicio, los estudiantes no se implican con la realidad del entorno, aunque sí hay un cambio de actitudes que suponga un compromiso por el cambio del contexto. La adhesión a contribuir a rehacer el pacto educativo es media.

Nivel III: cuando en el proyecto de Aprendizaje-Servicio, los estudiantes se implican con la realidad del entorno. Además, hay un cambio de actitudes que suponga un compromiso por el cambio del contexto. La adhesión a contribuir a rehacer el pacto educativo es alta.

Figura 1. Representación gráfica de la rúbrica.

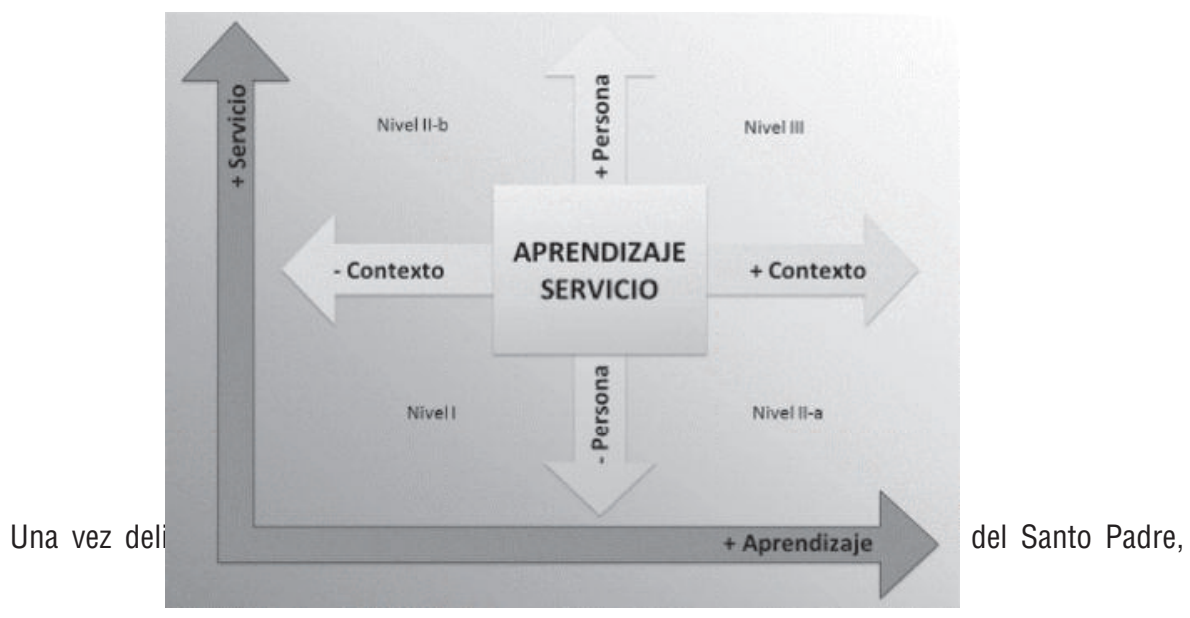


hemos seleccionado aquellas dimensiones que están más presentes y que por consiguiente son más significativas para la evaluación de proyectos Aprendizaje-Servicio. De esta manera tenemos 6 dimensiones: Cultura, Educación, Agentes sociales, Aprendizaje, Valores y Servicio. Cada dimensión está formada por 5 categorías pedagógicas. Destacamos pues las siguientes dimensiones y categorías:

Tabla 1

RÚBRICA PARA LA EVALUACIÓN DE EXPERIENCIAS DE APRENDIZAJE Y SERVICIO (REEAPS)

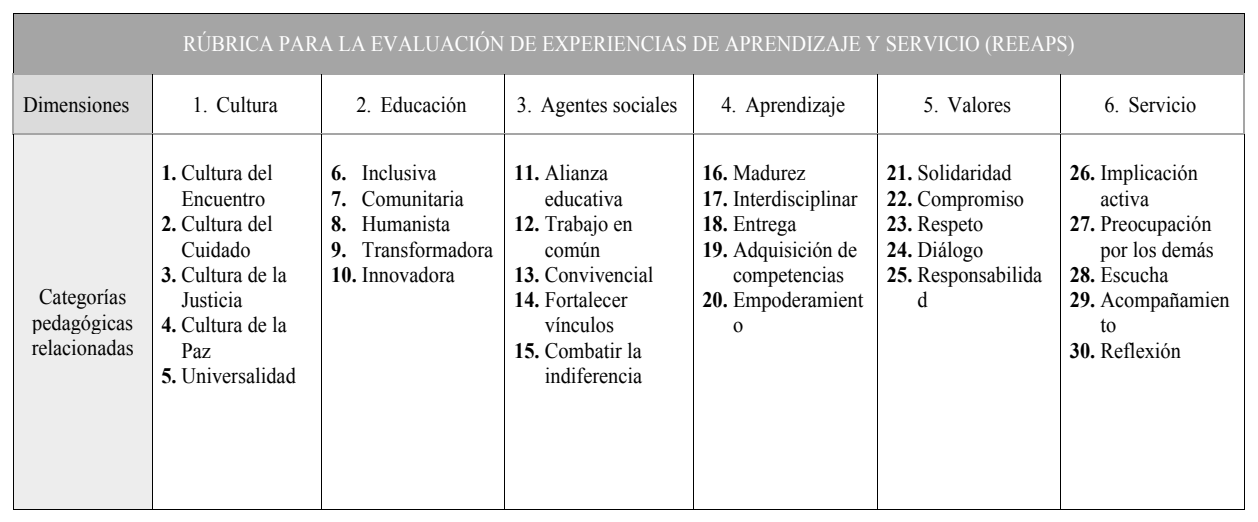

Las dimensiones y categorías han sido conceptualizadas teniendo presente tanto los escritos del Santo Padre como la literatura concerniente a la metodología Aprendizaje-Servicio. En este sentido, se van a presentar 6 dimensiones y cada una de ellas relacionadas con 5 categorías pedagógicas.

\section{REFERENCIAS BIBLIOGRÁFICAS}

Bergoglio, J. (1 de septiembre de 1999). Disertación de Mons. Jorge Mario Bergoglio en la sede de la Asociación Cristiana de empresarios, sobre el tema de la Educación. Recuperado el 12 de mayo de 2016, de Arzobispado de Buenos Aires:http://www.arzbaires.org.ar/inicio/homilias/homilias1999.htm\#Asociación Cristiana de empresarios.

Bergoglio, J. (9 de febrero de 2006). Palabras del Sr. Arzobispo Mario Bergolio en el Curso de Rectores. Recuperado el 15 de mayo de 2016, de Arzobispado de Buenos Aires: http://www.arzbaires.org.ar/inicio/homilias/homilias2006.htm

Francisco, PP. (7 de junio de 2013a). Discurso del Santo Padre Francisco a los estudiantes de las escuelas de los jesuitas de Italia y Albania. Recuperado el 7 de abril de 2016, de vaticano.va: https://w2.vatican.va/content/francesco/es/speeches/2013/june/documents/papa-francesco_20130607_scuole-gesuiti.html

Francisco, PP. (22 de julio de 2013b). Viaje Apostólico a Río de Janeiro con ocasión de la XXVIII Jornada Mundial de la Juventud. Ceremonia de bienvenida, discurso del Santo Padre. Recuperado el 3 de marzo de 2016, de vaticano.va: http://w2.vatican.va/content/francesco/es/travels/2013/outside/documents/papa-francescogmg-rio-de-janeiro-2013.html

Francisco, PP. (24 de noviembre de 2013c). Exhortación Apostólica Evangelii Gaudium del Santo 
Padre Francisco a los obispos a los presbíteros y diáconos a las personas consagradas y a los fieles laicos sobre el anuncio del evangelio en el mundo actual. Recuperado el 10 de abril de 2016, de vaticano.va: http://w2.vatican.va/content/francesco/es/apost_exhortations/documents/papa-francesco_esortazione-ap_20131124_evangelii-gaudium.html

Francisco, PP. (1 de enero de 2014a). Mensaje del Santo Padre Francisco para la celebración de la XLVII Jornada Mundial de la Paz. La fraternidad, fundamento y camino para la paz. Recuperado el 2016 de julio de 28, de vaticano.va: http://w2.vatican.va/content/francesco/es/ messages/peace/documents/papa-francesco_20131208_messaggio-xlvii-giornata-mondialepace-2014.html

Francisco, PP. (13 de febrero de 2014b). Discurso del Santo Padre Francisco a los participantes en la Plenaria de la Congregación para la Educación Católica. Recuperado el 19 de mayo de 2016, de vaticano.va: https://w2.vatican.va/content/francesco/es/speeches/2014/february/documents/papa-francesco_20140213_congregazione-educazione-cattolica.html

Francisco, PP. (28 de febrero de 2014c). Discurso del Santo Padre Francisco a los miembros de la Pontificia Comisión para América Latina. Recuperado el 13 de junio de 2016, de vaticano.va: https://w2.vatican.va/content/francesco/es/speeches/2014/february/documents/papa-francesc0_20140228_pontificia-commissione-america-latina.html

Francisco, PP. (10 de mayo de 2014d). Discurso del Santo Padre Francisco al mundo de la escuela italiana en la Plaza de San Pedro. Recuperado el 28 de junio de 2016, de vaticano.va: https://w2.vatican.va/content/francesco/es/speeches/2014/may/documents/papa-francesco_20140510_mondo-della-scuola.html

Francisco, PP. (4 de septiembre de 2014e). Discurso del Santo Padre Francisco a los participantes en el encuentro mundial de los directores de "Scholas Occurrentes". Recuperado el 4 de septiembre de 2016, de vaticano.va: https://w2.vatican.va/content/francesco/es/speeches /2014/september/documents/papa-francesco_20140904_direttori-scholas-occurrentes.html

Francisco, PP. (25 de noviembre de 2014f). Discurso del Santo Padre Francisco al Consejo de Europa Estrasburgo, Francia. Recuperado el 21 de junio de 2016, de vaticano.va: https://w2.vatican.va/content/francesco/es/speeches/2014/november/documents/papa-francesco_20141125_strasburgo-parlamento-europeo.html

Puig, J., Batlle, R., Bosch, C., \& Palos, J. (2007). Aprendizaje Servicio. Educar para la ciudadanía. Barcelona: Octaedro.

Puig (coord.), J., Batlle, R., Bosch, C., de la Cerda, M., Climent, T., Gijón, M., y otros. (2009). Aprendizaje-Servicio (ApS). Educación y compromiso cívico. Barcelona: Graó.

Rubin, S., \& Ambrogetti, F. (2015). El Papa Francisco. Conversaciones con Jorge Bergoglio. Barcelona: Ediciones B.

Tapia, N. (2004). Aprendizaje y servicio solidario. Madrid: CCS. 